\title{
Clinical Interventions to Prevent Tumour Lysis Syndrome in Hematologic Malignancy: A Multisite Retrospective Chart Review
}

\author{
Sarah McKenna, Alexandra Cheung, Amanda Wolfe, Brenda L Coleman, Michael E Detsky, \\ Laveena Munshi, Dawn Maze, and Lisa Burry
}

\begin{abstract}
Background: Tumour lysis syndrome (TLS) occurs when lysis of malignant cells causes electrolyte disturbances and potentially organ dysfunction. Guidelines recommending preventive therapy according to TLS risk are based on low-quality evidence.

Objectives: The primary objective was to characterize utilization of TLS preventive strategies through comprehensive description of current practice. Secondary objectives were to determine TLS incidence, to compare use of preventive strategies among intermediate- and high-risk patients, and to describe TLS treatment strategies.

Methods: This retrospective chart review examined data for patients with newly diagnosed hematologic malignancy who were admitted to an oncology centre and/or affiliated intensive care unit between October 2015 and September 2016 in Toronto, Ontario, Canada.

Results: Fifty-eight patients (29 at intermediate risk, 29 at high risk) were eligible for inclusion. Use of preventive allopurinol, IV bicarbonate, and furosemide was similar between groups. Rasburicase was more frequently used for high-risk patients $(3 \%$ [1/29] of intermediate-risk patients versus $36 \%$ [9/25] of high-risk patients; $p=0.003)$. In $4(14 \%)$ of the intermediate-risk patients and $2(8 \%)$ of the high-risk patients, TLS developed during the admission. TLS was observed in $10 \%(1 / 10)$ of patients who received preventive rasburicase and $11 \%(5 / 44)$ of those who did not $(p>0.99)$, and in $9 \%$ ( $4 / 45)$ of patients who received preventive IV bicarbonate and $25 \%(2 / 8)$ of those who did not $(p=0.22)$. Treatment strategies included rasburicase, IV bicarbonate, furosemide, and renal replacement therapy.
\end{abstract}

Conclusions: In this retrospective chart review, rasburicase was more commonly used for high-risk patients, whereas the use of other agents was similar between risk groups. This pattern of use is inconsistent with guidelines, which recommend that all high-risk patients receive rasburicase. There was no difference in TLS incidence between patients who did and did not receive preventive rasburicase or IV bicarbonate. Further prospective studies are needed to inform management of patients with malignancies who are at intermediate or high risk of TLS.

Keywords: tumour lysis syndrome, rasburicase, bicarbonate, allopurinol

\section{RÉSUMÉ}

Contexte : Le syndrome de lyse tumorale (SLT) se produit lorsque la lyse de cellules malignes provoque des perturbations électrolytiques et la dysfonction potentielle d'un organe. Les lignes directrices préconisant une thérapie préventive basée sur le risque de SLT se fondent sur des éléments de preuve de piètre qualité.

Objectifs : L'objectif principal consistait à décrire l'adoption des stratégies de prévention du SLT en décrivant précisément la pratique actuelle. Les objectifs secondaires consistaient, quant à eux, à déterminer l'incidence du SLT, à comparer l'utilisation des stratégies de prévention pour les patients présentant un risque élevé et moyen et à décrire les stratégies de traitement du SLT.

Méthodes : Cet examen rétrospectif a permis d'examiner les données de patients ayant récemment reçu un diagnostic d'hémopathie maligne et ayant été admis dans un centre d'oncologie ou une unité de soins intensifs affiliée, entre octobre 2015 et septembre 2016 à Toronto (Ontario), au Canada.

Résultats : Cinquante-huit patients ( 29 présentant un risque moyen et 29 un risque élevé) étaient admissibles. L'utilisation d'allopurinol à titre préventif, de bicarbonate par voie intraveineuse et de furosémide était similaire d'un groupe à l'autre. Le rasburicase était plus fréquemment utilisé pour les patients présentant un risque élevé (3\% [1/29] de patients présentant un risque moyen contre $36 \%$ [9/25] de patients présentant un risque élevé; $p=0.003)$. Quatre (14\%) patients présentant un risque moyen et deux $(8 \%)$ présentant un risque élevé ont développé un SLT pendant l'admission. Le SLT a été observé chez $10 \%$ (1/10) des patients ayant reçu du rasburicase à titre préventif et chez $11 \%(5 / 44)$ des patients qui n'en avaient pas reçu ( $p>0,99)$; il a aussi été observé chez $9 \%$ (4/45) des patients ayant reçu du bicarbonate par voie intraveineuse à titre préventif et chez $25 \%(2 / 8)$ des patients qui n'en avaient pas reçu $(p=0.22)$. Les stratégies de traitement comprenaient le rasburicase, le bicarbonate par voie intraveineuse, le furosémide et la thérapie de remplacement rénal.

Conclusions : Dans cet examen rétrospectif des dossiers, l'usage du rasburicase était plus fréquent pour les patients présentant un risque élevé, tandis que celui d'autres agents était similaire entre les groupes à risque. Ce schéma d'utilisation n'est pas conforme aux lignes directrices, qui 


\section{Can J Hosp Pharm. 2019;72(6):435-45}

recommandent que tous les patients présentant un risque élevé reçoivent du rasburicase. Aucune différence n'est apparue dans l'incidence du SLT parmi les patients ayant reçu du rasburicase ou du bicarbonate par voie intraveineuse à titre préventif et parmi ceux qui n'en avaient pas reçu. Davantage d'études prospectives sont nécessaires pour mieux connaitre la gestion des patients à haut risque ou ceux qui présentent des risques moyens de SLT, mais qui ont des malignités.

Mots clés : syndrome de lyse tumorale, rasburicase, bicarbonate, allopurinol

\section{INTRODUCTION}

$\mathrm{T}$ umour lysis syndrome (TLS) is a medical emergency in which massive lysis of malignant cells upon exposure to cytotoxic therapy leads to metabolic derangements and organ dysfunction, including renal failure, seizures, and cardiac arrhythmias. ${ }^{1}$ Acute lymphoblastic leukemia, acute myeloid leukemia, and aggressive non-Hodgkin lymphoma pose the greatest risk of TLS; however, other cancers with specific tumour-related factors (such as high sensitivity to cytotoxic therapy, high tumour burden, or high rate of proliferation) are also susceptible. ${ }^{1,2}$ TLS may also occur spontaneously in the absence of cytotoxic therapy, but is more commonly induced by initial exposure to a cytotoxic agent such as hydroxyurea, steroid, or definitive chemotherapy. ${ }^{3}$

TLS is classified as either laboratory or clinical. Laboratory TLS is defined as 2 or more of the following within 3 days before or 7 days after exposure to cytotoxic therapy: uric acid $\geq 476 \mu \mathrm{mol} / \mathrm{L}$ or $25 \%$ increase from baseline, potassium $\geq 6.0 \mathrm{mmol} / \mathrm{L}$ or $25 \%$ increase from baseline, phosphate $\geq 1.45 \mathrm{mmol} / \mathrm{L}$ or $25 \%$ increase from baseline, or calcium $\leq 1.75 \mathrm{mmol} / \mathrm{L}$ or $25 \%$ decrease from baseline. Clinical TLS is defined as the occurrence of laboratory TLS plus 1 of the following: creatinine $\geq 1.5$ times the institutional upper limit of normal, seizure, or cardiac arrhythmia/sudden death. ${ }^{1}$

A risk classification system, which stratifies patients as having low, intermediate, or high risk of TLS according to their type or stage of cancer, white blood cell count, and serum lactate dehydrogenase at time of presentation, is used to guide preventive therapy. ${ }^{3}$ Prevention of TLS involves maintenance of adequate hydration and administration of medications to decrease serum uric acid, with the intention of preventing renal failure. Guidelines recommend that low-risk patients be actively monitored with careful attention to fluid status, and that intermediate-risk patients receive initial management with IV fluids and allopurinol to decrease production of uric acid, starting 1 to 2 days before definitive chemotherapy and continuing for up to 7 days afterward. ${ }^{2,4}$ Rasburicase, a recombinant urate-oxidase enzyme that catalyzes the metabolism of poorly soluble uric acid to the more-soluble allantoin, is recommended for intermediate-risk patients if hyperuricemia develops despite prophylaxis with allopurinol. ${ }^{2,4}$ High-risk patients should receive initial management with IV fluids and rasburicase. ${ }^{2,4}$

Treatment of established TLS involves interventions to target each laboratory abnormality, as dictated by its severity. Treatment may include IV administration of calcium to replenish serum calcium and to prevent arrhythmia, IV administration of insulin or sodium bicarbonate to induce intracellular potassium shift, administration of rasburicase to decrease serum uric acid, and administration of phosphate binders to decrease serum phosphate. In severe cases that have not responded to initial interventions, renal replacement therapy (RRT) may be required. ${ }^{1}$ Electrocardiogram findings, urine output, and electrolytes should be assessed every 4 to $6 \mathrm{~h}$, and admission to an intensive care unit (ICU) may be required for patients with no response to initial interventions. ${ }^{1,2}$ The authors' institutions (described below) do not have a standardized approach to risk stratification for patients who present with malignancy and with intermediate or high risk of TLS, because there are no high-quality studies to inform such guidelines. As a result, there is likely variability in the approach to TLS prevention across these institutions.

The primary objective of this study was to characterize utilization of preventive strategies for patients with newly diagnosed hematologic malignancies who are at intermediate or high risk of TLS through comprehensive description of current practice (as one of our quality improvement initiatives). Secondary objectives were to determine TLS incidence, to compare use of preventive strategies among intermediate- and high-risk patients, and to describe TLS treatment strategies.

\section{METHODS}

\section{Study Design}

This multisite, retrospective chart review of patients admitted to Princess Margaret Cancer Centre (PM) and/or the affiliated ICU at Mount Sinai Hospital (MSH) in Toronto, Ontario, Canada, was an initial phase of the organizations' oncology quality improvement initiatives. Electronic medical records were reviewed alphabetically by last name to identify eligible patients admitted to either institution within the 1-year study period of October 1, 2015, to September 30, 2016. On the basis of historical admission 
information for patients with hematologic malignancy at risk of TLS, we estimated that a 1-year time frame would provide an adequate sample size to thoroughly describe current practice.

This study was approved by the research ethics boards at PM and $\mathrm{MSH}$, both of which waived the need for informed consent. The research was conducted in accordance with the Helsinki Declaration. A data transfer agreement was completed to permit transfer of information between the institutions.

\section{Study Participants}

Potential participants were identified using International Classification of Diseases and Related Health Problems, 10th revision (ICD-10) codes for malignancies of interest or for TLS (Table 1). Patients were considered eligible if the reason for admission to the ICU at MSH or to PM was treatment of a newly diagnosed malignancy or treatment of suspected TLS. The TLS risk stratification criteria previously defined by an international expert panel were then applied to stratify patients as having low, intermediate, or high risk for TLS according to the type of malignancy, serum white blood cell count, and serum lactate dehydrogenase at the time of presentation (Table 2). ${ }^{3,5}$ Patients were excluded from this study if they had low risk for TLS or if the reason for admission was management of a recurrent malignancy. We excluded low-risk patients because it was likely that use of preventive strategies in this group would be too infrequent for adequate characterization given that monitoring is the recommended management strategy for these patients. ${ }^{2,4} \mathrm{We}$ excluded patients with recurrent malignancy because we suspected that the risk of TLS among those experiencing relapse or refractory malignancy might have been affected by confounding factors, including the TLS preventive strategies employed during previous admissions.
Table 1. Codes from the International Classification of Diseases and Related Health Problems, 10th Revision (ICD-10) Used to Identify Potential Participants

\begin{tabular}{lc} 
Condition & ICD-10 code \\
\hline Acute myeloblastic leukemia & $\mathrm{C} 92.0$ \\
Acute promyelocytic leukemia & $\mathrm{C} 92.4$ \\
Acute myelomonocytic leukemia & $\mathrm{C} 92.5$ \\
Acute myeloid leukemia with 11q23-abnormality & $\mathrm{C} 92.6$ \\
Acute myeloid leukemia with multilineage & $\mathrm{C} 92.8$ \\
$\quad$ dysplasia & $\mathrm{C} 93.0$ \\
Acute monoblastic/monocytic leukemia & $\mathrm{C} 94.2$ \\
Acute megakaryoblastic leukemia & $\mathrm{C} 91.0$ \\
Acute lymphoblastic leukemia & $\mathrm{C} 83.3$ \\
Diffuse large B-cell lymphoma & $\mathrm{C} 83.5$ \\
Lymphoblastic (diffuse) lymphoma & $\mathrm{C} 85.2$ \\
Mediastinal (thymic) large B-cell lymphoma & $\mathrm{C} 83.7$ \\
Burkitt lymphoma & $\mathrm{C} 91.8$ \\
Mature B-cell leukemia Burkitt-type & $\mathrm{E} 88.3$ \\
Tumour lysis syndrome & \\
\hline
\end{tabular}

\section{Definitions}

We defined laboratory TLS as 2 or more of the following at any point within the data collection period: uric acid $\geq 476 \mu \mathrm{mol} / \mathrm{L}$, potassium $\geq 6.0 \mathrm{mmol} / \mathrm{L}$, phosphate $\geq 1.45 \mathrm{mmol} / \mathrm{L}$, or calcium $\leq 1.75 \mathrm{mmol} / \mathrm{L}$. ${ }^{1}$ We did not account for changes from baseline, because baseline values could not be obtained for all patients. TLS was defined as spontaneous if the patient had not been exposed to any cytotoxic therapies (such as chemotherapy, steroids, or hydroxyurea) immediately before admission.

We defined clinical TLS as the presence of laboratory TLS plus 1 of the following: renal failure (defined as serum creatinine $\geq 1.5$ times the institutional upper limit of normal), seizure, or

Table 2. Hematologic Malignancies of Interest and Associated Characteristics Used as Inclusion Criteria ${ }^{3,5}$

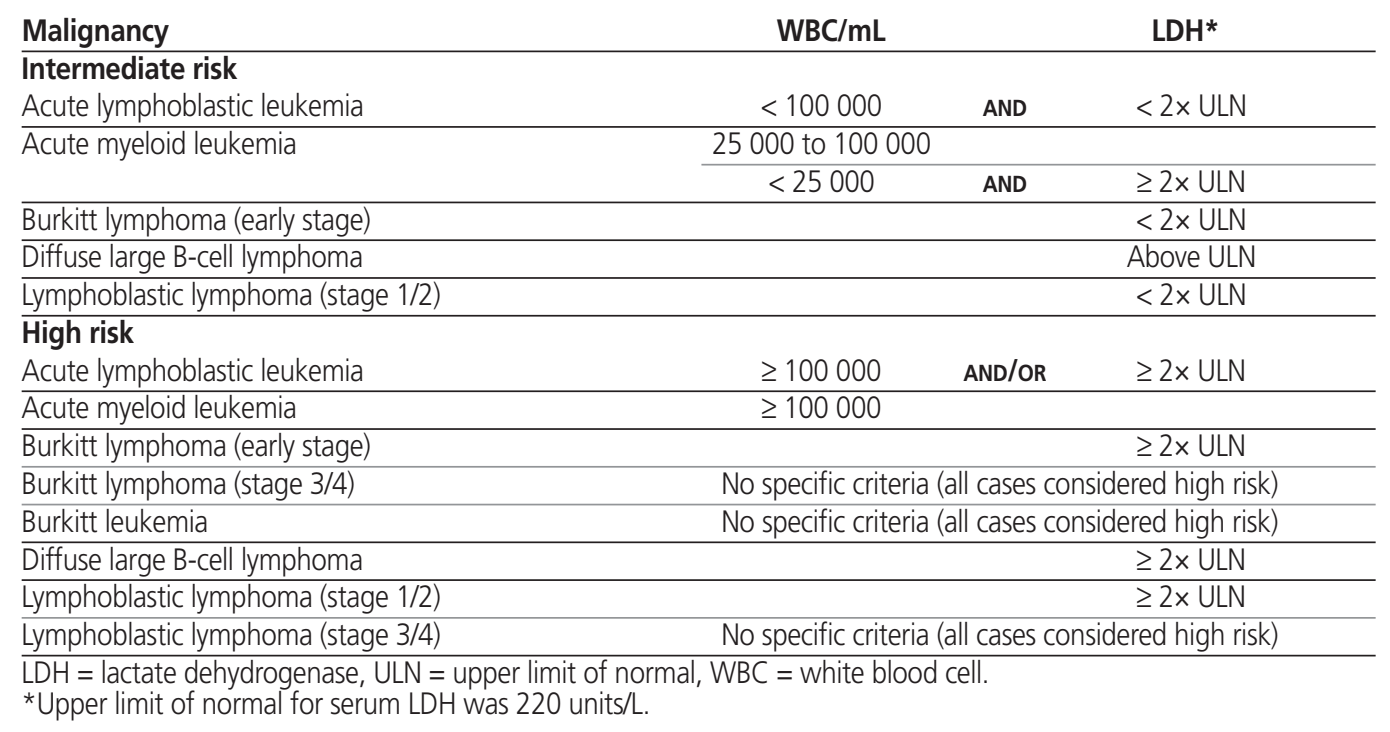


cardiac arrhythmia. ${ }^{1}$ We did not include death as a marker for clinical TLS because death could not be definitively attributed to TLS alone in the context of a retrospective study. Clinical outcome data were obtained from clinical notes and discharge summaries.

\section{Study Outcomes}

The primary outcome was a description of current utilization of TLS preventive strategies, as indicated by the proportion of intermediate- and high-risk patients who received specific preventive strategies during admission, namely allopurinol, rasburicase, IV fluids (with or without sodium bicarbonate), and furosemide. The difference between the 2 risk groups in the proportions of patients who received each intervention was a secondary outcome. The 2 additional secondary outcomes were determination of TLS incidence and description of treatment strategies employed for patients who presented with TLS and for those in whom TLS developed during the admission.

\section{Data Collection}

For patients who received chemotherapy within 7 days of admission, data were collected from the day of admission up to
7 days after initiation of chemotherapy. For patients who did not receive chemotherapy within 7 days of admission, data were collected for the first 7 days of the hospital stay. For patients who were transferred between PM and MSH, data were collected from both institutions if the transfer occurred within the data collection window. Data were collected by trained investigators at both sites and were audited by a single investigator (S.M.).

\section{Statistical Analysis}

Data were analyzed using the $\chi^{2}$ and Fisher exact tests for categorical variables. All of the tests were 2 -sided, and the results were considered statistically significant if $p$ was less than 0.05 . Statistical analyses were performed using Stata software, version 11.2 (StataCorp LLC, College Station, Texas).

\section{RESULTS}

\section{Study Population}

Of 313 patients screened, 58 met the inclusion criteria (Figure 1). Twenty-nine patients $(50 \%)$ were deemed to be at intermediate risk of TLS and 29 (50\%) were deemed to be high

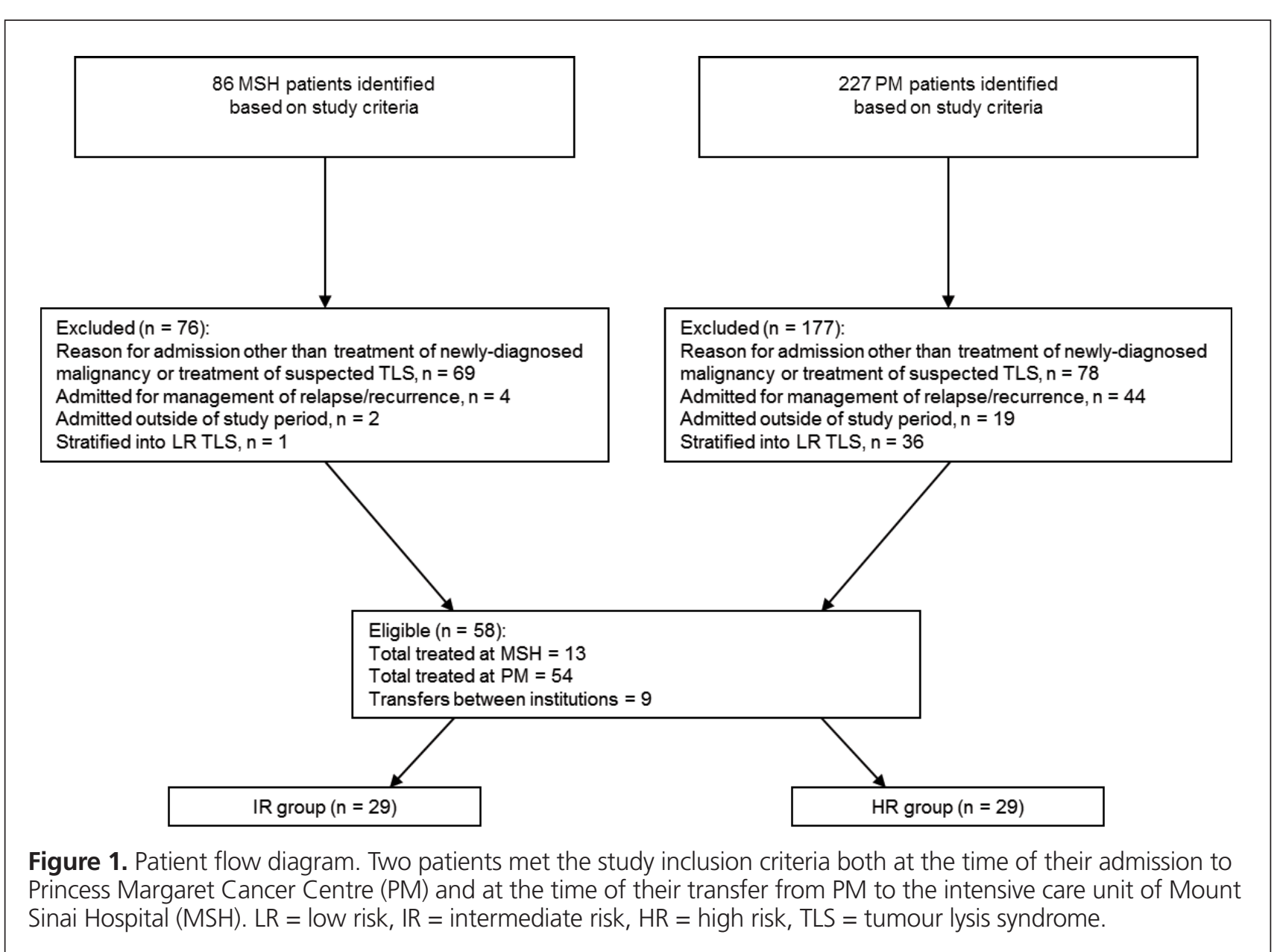


This single copy is for your personal, non-commercial use only.

For permission to reprint multiple copies or to order presentation-ready copies for distribution, contact CJHP at publications@cshp.pharmacy

risk. Two patients met the study inclusion criteria both at the time of their admission to PM and at the time of their transfer to MSH from PM; for each of these patients, the data for the 2 admissions were merged. The study groups were not significantly different at baseline (Table 3). Most of the patients (76\% [44/58]) presented with either acute lymphoblastic leukemia or acute myeloid leukemia, and most (91\% [53/58]) received chemotherapy within 7 days of admission.

Table 3. Demographic and Baseline Clinical Characteristics

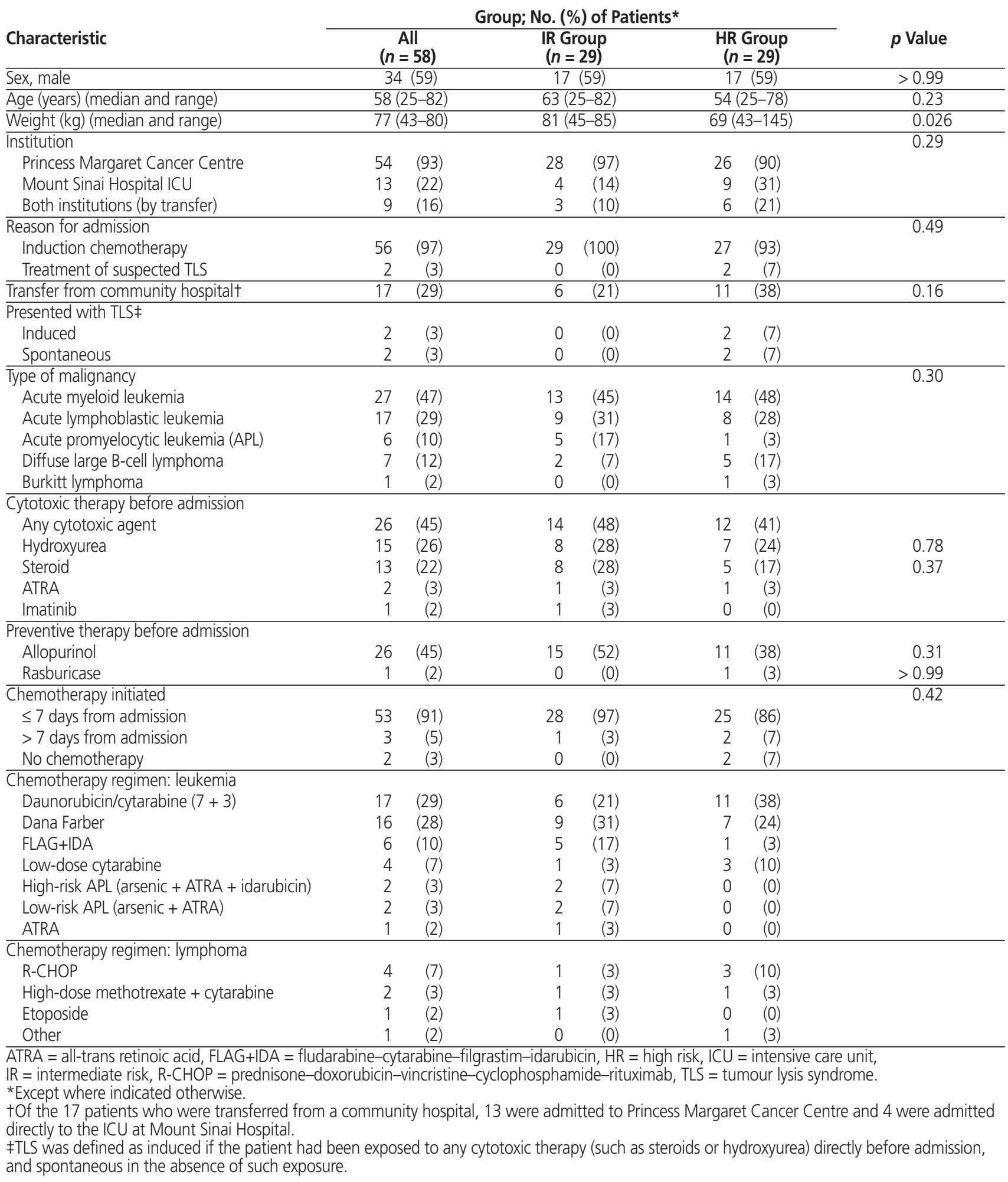




\section{Use of Preventive Strategies}

Use of certain preventive strategies did not differ between the intermediate- and high-risk groups (Table 4): allopurinol, 90\% versus $92 \%$ ( $p>0.99$ ); IV fluids, $83 \%$ with bicarbonate and $14 \%$ without bicarbonate versus $84 \%$ with bicarbonate and $16 \%$ without bicarbonate ( $p>0.99$ ); and furosemide, $14 \%$ versus $12 \%$ $(p>0.99)$. Use of preventive rasburicase was significantly higher in the high-risk group ( $3 \%$ versus $36 \% ; p=0.003$ ). For all patients who received allopurinol for TLS prevention, the dosage was $300 \mathrm{mg}$ once daily. For all patients who received rasburicase for TLS prevention, the dose was $4.5 \mathrm{mg}$. Of the 10 patients who received preventive rasburicase, 4 had serum uric acid at or above $476 \mu \mathrm{mol} / \mathrm{L}$.

\section{Incidence of TLS}

\section{All Participants}

There were 10 cases of TLS in total: for 6 patients, TLS developed during the hospital stay, and for 4 patients, TLS was present at the time of admission. Among the 54 patients who did not have TLS at the time of presentation, 29 were at intermediate risk and 25 at high risk. Among those at intermediate risk, TLS occurred in 4 patients and did not occur in the remaining 25 patients; among those at high risk, TLS occurred in 2 patients and did not occur in the remaining 23 patients (Figure 2). Of the 4 intermediate-risk patients in whom laboratory TLS occurred, 3 also experienced clinical TLS. Of the 2 high-risk patients in whom laboratory TLS occurred, both also experienced clinical TLS. In 1 high-risk patient, laboratory TLS developed 3 days before initiation of definitive chemotherapy; for the remaining 5 patients with laboratory TLS, it occurred after chemotherapy initiation. Laboratory abnormalities observed in the 10 patients with TLS are shown in Table 5.

All 4 patients who presented with TLS at the time of admission were at high risk and presented with clinical TLS. Three of these patients had acute myeloid leukemia, and 1 had diffuse large B-cell lymphoma.

Abnormal laboratory findings were more common in high-risk than in intermediate-risk patients. Several patients had an abnormal result for only 1 laboratory parameter and thus did not satisfy the criteria for laboratory TLS (Table 6).

Table 4. Utilization of Preventive Strategies According to Risk of Tumour Lysis Syndrome

\begin{tabular}{lrrrrr} 
& \multicolumn{2}{c}{ Risk Level; No. (\%) of Patients } & \\
\cline { 2 - 4 } Preventive Strategy & $\begin{array}{c}\text { Intermediate Risk } \\
(\boldsymbol{n}=\mathbf{2 9 )}\end{array}$ & $\begin{array}{c}\text { High Risk } \\
(\boldsymbol{n}=\mathbf{2 5})\end{array}$ & $\boldsymbol{p}$ Value \\
\hline Allopurinol & 26 & $(90)$ & 23 & $(92)$ & $>0.99$ \\
\hline Rasburicase & 1 & $(3)$ & 9 & $(36)$ & 0.003 \\
\hline IV fluids & 24 & $(83)$ & 21 & $(84)$ & $>0.99$ \\
$\quad$ With bicarbonate & 4 & $(14)$ & 4 & $(16)$ & \\
$\quad$ Without bicarbonate & 4 & $(14)$ & 3 & $(12)$ & $>0.99$ \\
\hline Furosemide & & & &
\end{tabular}

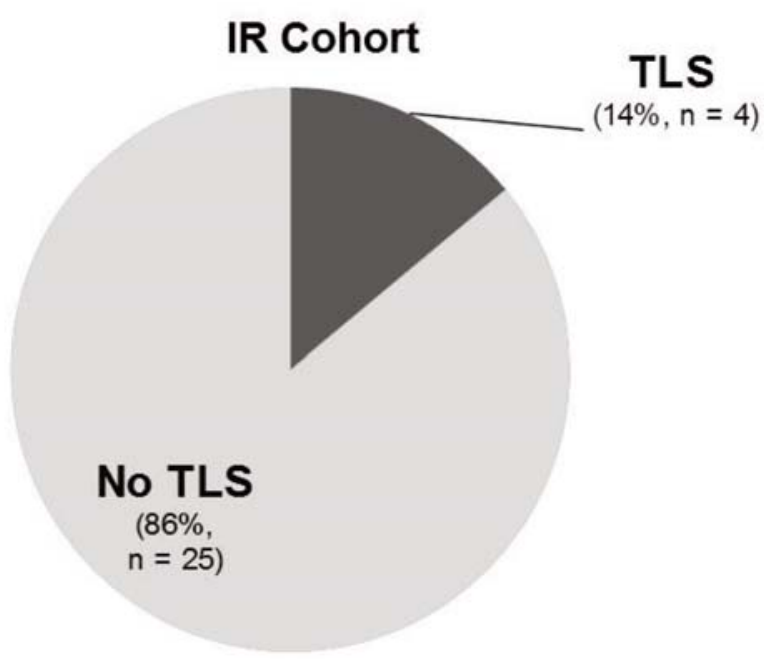

HR Cohort

TLS $(8 \%, n=2)$

Figure 2. Occurrence of tumour lysis syndrome (TLS) among patients who did not present with TLS. $\mathrm{IR}=$ intermediate risk, $\mathrm{HR}=$ high risk. 
Table 5. Incidence of Abnormal Laboratory Values in Patients with TLS

\begin{tabular}{lcrcr} 
& \multicolumn{4}{c}{ Occurrence of TLS; No. (\%) of Patients } \\
\cline { 2 - 5 } Abnormality & $\begin{array}{c}\text { Patient Presented } \\
\text { with TLS }(\boldsymbol{n}=\mathbf{4})\end{array}$ & $\begin{array}{c}\text { TLS Developed } \\
\text { in Hospital }(\boldsymbol{n}=\mathbf{6})\end{array}$ \\
\hline Uric acid $\geq 476 \mu \mathrm{mol} / \mathrm{L}$ & 4 & $(100)$ & 4 & $(67)$ \\
Potassium $\geq 6 \mathrm{mmol} / \mathrm{L}$ & 1 & $(25)$ & 2 & $(33)$ \\
Phosphate $\geq 1.45 \mathrm{mmo} / \mathrm{L}$ & 4 & $(100)$ & 6 & $(100)$ \\
Calcium $\leq 1.75 \mathrm{mmol} / \mathrm{L}$ & 1 & $(25)$ & 4 & $(67)$ \\
\hline
\end{tabular}

TLS = tumour lysis syndrome.

Table 6. Incidence of Abnormal Laboratory Values According to Risk Level

\begin{tabular}{lrrrrr} 
& \multicolumn{3}{c}{ Risk Level; No. (\%) of Patients } \\
\cline { 2 - 6 } Abnormality & \multicolumn{3}{c}{$\begin{array}{c}\text { Intermediate Risk } \\
(\boldsymbol{n}=\mathbf{2 9})\end{array}$} & \multicolumn{2}{c}{$\begin{array}{c}\text { High Risk } \\
(\boldsymbol{n}=\mathbf{2 9})\end{array}$} \\
\hline Uric acid $\geq 476 \mu \mathrm{mol} / \mathrm{L}$ & 4 & $(14)$ & 9 & $(31)$ \\
Potassium $\geq 6 \mathrm{mmo} / \mathrm{L}$ & 1 & $(3)$ & 2 & $(7)$ \\
Phosphate $\geq 1.45 \mathrm{mmol} / \mathrm{L}$ & 21 & $(72)$ & 24 & $(83)$ \\
Calcium $\leq 1.75 \mathrm{mmol} / \mathrm{L}$ & 3 & $(10)$ & 4 & $(14)$ \\
\hline
\end{tabular}

\section{Table 7. Characteristics of Patients with Clinical Tumour Lysis Syndrome (CTLS) Who Received or Did Not Receive Renal Replacement Therapy (RRT)*}

\begin{tabular}{|c|c|c|c|}
\hline $\begin{array}{l}\text { Patient Designation and } \\
\text { Categorization }\end{array}$ & $\begin{array}{c}\text { Electrolyte } \\
\text { Abnormalitiest }\end{array}$ & $\begin{array}{c}\text { Decreased Urine } \\
\text { Output‡ }\end{array}$ & $\begin{array}{l}\text { Serum Creatinine } \\
\geq 1.5 \text { ULN§ }\end{array}$ \\
\hline \multicolumn{4}{|l|}{$\begin{array}{l}\text { CTLS developed during admission } \\
\text { RRT initiated }\end{array}$} \\
\hline $1-010$ & Yes (K 6.8 mmol/L) & - & No \\
\hline $1-051$ & Yes (K $6.8 \mathrm{mmol} / \mathrm{L})$ & - & Yes \\
\hline $1-057$ & Yes & Sudden decrease & Yes \\
\hline \multicolumn{4}{|l|}{ RRT not initiated } \\
\hline $1-011$ & Yes & - & Yes \\
\hline $1-040$ & Yes & - & Yes \\
\hline \multicolumn{4}{|l|}{ Patient presented with CTLS** } \\
\hline $1-021$ & Yes & - & Yes \\
\hline $1-038$ & Yes (K $6.6 \mathrm{mmol} / \mathrm{L})$ & - & Yes \\
\hline $1-052$ & Yes & - & Yes \\
\hline $1-058$ & Yes & - & Yes \\
\hline \multicolumn{4}{|c|}{$\begin{array}{l}\text { K= potassium, ULN = upper limit of normal. } \\
{ }^{*} \text { Characteristics shown in this table (electrolyte abnormalities, decrease in urine output, serum creatinine level) were } \\
\text { considered consistent with clinical tumour lysis syndrome only if they occurred simultaneously. } \\
\text { †Elevated serum potassium, elevated serum uric acid, elevated serum phosphate, or decreased serum calcium. If serum } \\
\text { potassium was elevated, the value is specifically indicated. } \\
\text { fUrine output was recorded for patients admitted to the intensive care unit at Mount Sinai Hospital, but not for those } \\
\text { admitted to Princess Margaret Cancer Centre. Therefore, this variable could not be reported for some patients. } \\
\text { §Upper limit of normal for serum creatinine was defined as } 80 \mu \mathrm{mol} / \mathrm{L} \text { for women and } 105 \mu \mathrm{mol} / \mathrm{L} \text { for men. } \\
\star \star * \text { None of the patients who presented with tumour lysis syndrome received renal replacement therapy. }\end{array}$} \\
\hline
\end{tabular}

Each of the 5 patients in whom clinical TLS developed during admission had documented acute kidney injury, and 3 of these received RRT. Each of these 5 patients had received chemotherapy within 7 days of admission. One of these 5 patients had a documented cardiac arrhythmia (serum potassium level was normal throughout the admission). All 4 patients in whom clinical TLS was present at the time of admission had documented acute kidney injury, although none received RRT. One of these patients had a documented cardiac arrhythmia (serum potassium on admission was $6.6 \mathrm{mmol} / \mathrm{L}$ ). Characteristics of patients with clinical TLS who did and did not receive RRT are shown in Table 7.

\section{Patients Who Received Preventive Rasburicase and/or IV Sodium Bicarbonate}

Of the 10 patients who received preventive rasburicase, $1(10 \%)$ experienced clinical TLS. Of the 44 patients who did not receive preventive rasburicase, 5 (11\%) experienced laboratory TLS and 4 (9\%) also experienced clinical TLS. There was no statistically significant difference in incidence of TLS between those who did and did not receive preventive rasburicase (Table 8).

Of the 45 patients who received preventive IV sodium bicarbonate, 4 (8\%) experienced laboratory TLS, and $3(6 \%)$ also 
experienced clinical TLS. Of the 8 patients who did not receive preventive IV sodium bicarbonate, 2 (25\%) experienced clinical TLS. There was no statistically significant difference in incidence of TLS between those who did and did not receive preventive IV sodium bicarbonate (Table 8).

\section{Treatment Strategies}

All 10 patients with TLS (either present at time of admission $[n=4]$ or occurring during the hospital stay $[n=6])$ required interventions for treatment of TLS.

Of the 4 patients who presented with TLS, all had serum uric acid at or above $476 \mu \mathrm{mol} / \mathrm{L}$, and all received rasburicase. Two of these patients received a single dose of rasburicase, and the other 2 patients received 2 doses. Of these latter 2 patients (who received 2 doses), only 1 had serum uric acid at or above $476 \mu \mathrm{mol} / \mathrm{L}$ after the first dose. All 4 patients who presented with TLS received IV fluids containing sodium bicarbonate. RRT was not initiated for any of these patients (Figure 3).

Of the 6 patients in whom TLS developed during admisson, 4 had serum uric acid at or above $476 \mu \mathrm{mol} / \mathrm{L}$, yet only 1 received rasburicase. Four of these 6 patients received IV fluids containing sodium bicarbonate, whereas the remaining 2 received IV fluids without sodium bicarbonate. RRT was initiated for 3 of these 6 patients.

\section{Admission to ICU}

Fifty-four of the 58 patients in this study were admitted directly to PM, and 9 of these (3 at intermediate risk and 6 at high risk) were later transferred to MSH for ICU care. In 5 of the transferred patients, the development of TLS occurred directly before transfer ( 4 cases of induced TLS and 1 case of spontaneous TLS). Three of the transferred patients died in the ICU; all had experienced induced TLS on the day of transfer. RRT had been initiated for 2 of these patients but not for the third patient, despite severe electrolyte abnormalities.

The remaining 4 patients were admitted directly to the ICU at MSH (3 at high risk, 1 at intermediate risk). Chemotherapy was not initiated for 2 of these patients, and both died shortly after admission. Chemotherapy was initiated for the other 2 patients. One of these patients (who was at high risk) had acute myeloid leukemia and died on the day of chemotherapy initiation; this patient had not experienced TLS at any point. The other patient (who was at intermediate risk) also had acute myeloid leukemia, experienced TLS after initiation of chemotherapy, and required RRT but did not die.

\section{DISCUSSION}

In this multicentre review of TLS preventive strategies, we observed variability in the approach to TLS prevention between intermediate- and high-risk patients. Preventive rasburicase was used more frequently for high-risk patients, although not all patients in this risk category received the intervention. Use of allopurinol, IV fluids (with or without bicarbonate), and furosemide was similar between the intermediate- and high-risk groups.

\section{Urinary Alkalinization for TLS Prevention}

In this study, $83 \%$ of patients received IV fluids containing sodium bicarbonate for TLS prevention, a practice that clinical guidelines recommend against. ${ }^{2,4}$ Urinary alkalinization increases uric acid solubility, decreasing precipitation of uric acid crystals in the renal tubules and preventing any obstruction that might otherwise ensue. ${ }^{6}$ However, alkalinization does not prevent deposition of calcium phosphate crystals; rather, the solubility of calcium phosphate decreases with increasing $\mathrm{pH}$, which increases the risk of obstruction and acute kidney injury. ${ }^{7}$ Additionally,

Table 8. Incidence of Tumour Lysis Syndrome in Relation to Receipt of Rasburicase and/or IV Fluids Containing Sodium Bicarbonate for Prevention*

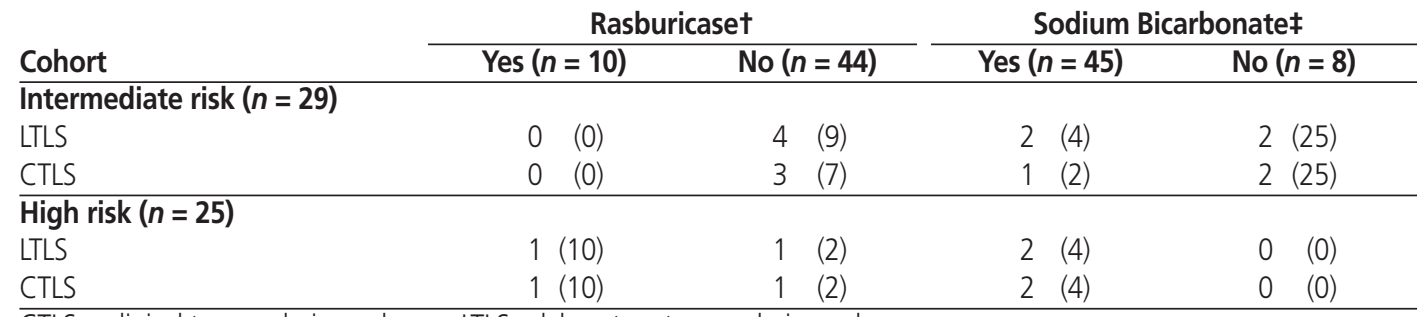

CTLS = clinical tumour lysis syndrome, LTLS = laboratory tumour lysis syndrome.

* Data presented in this table are for the 54 patients who did not have tumour lysis syndrome at the time of presentation; data for the 4 patients who had this condition on presentation are excluded. Data are shown as number (\%) of patients, based on $n$ value at the top of each column.

tThere was no significant difference in the occurrence of TLS among patients who did and did not receive preventive rasburicase, for both risk categories combined $(p>0.99)$.

¥Sodium bicarbonate data are shown for a total of 53 patients (1 patient did not receive any IV fluids). There was no significant difference in the occurrence of TLS among patients who did and did not receive preventive sodium bicarbonate, for both risk categories combined $(p=0.22)$. 


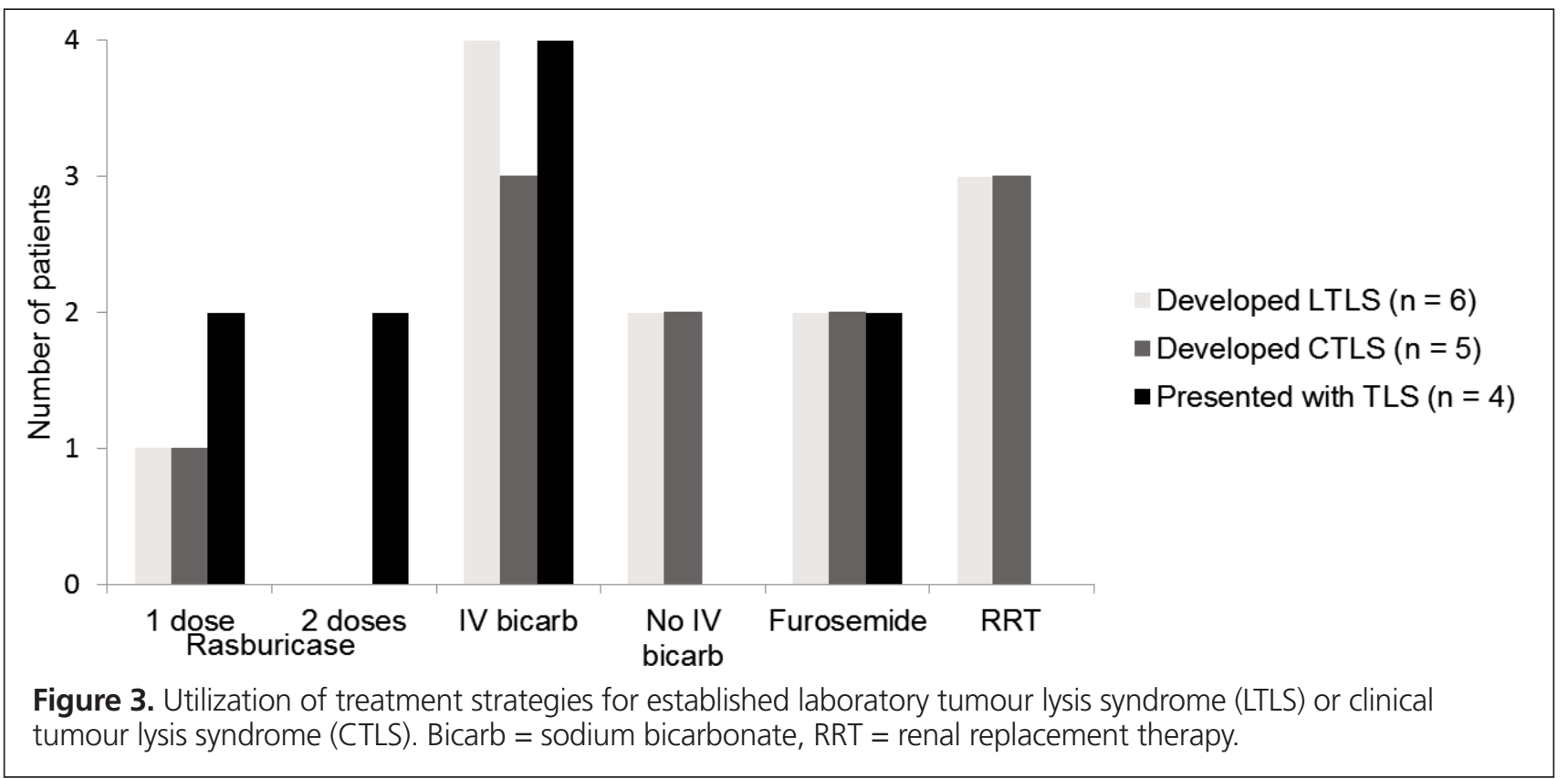

alkalinization does not increase the solubility of the uric acid precursors xanthine and hypoxanthine. Because the levels of these precursors are elevated in patients receiving allopurinol, this represents a potential additional cause of acute kidney injury. ${ }^{6}$ Administration of sodium bicarbonate has also been shown to increase carbon dioxide production and to worsen respiratory distress in susceptible patients, which may warrant ICU admission. ${ }^{8-10}$ Efficacy of the intervention in preventing acute nephropathy has not been demonstrated, and the evidence is limited to a single animal study in which urinary alkalinization was not effective in preventing intrarenal deposition of urate. ${ }^{11}$ Despite the lack of evidence for efficacy and numerous concerns about potential harm, administration of sodium bicarbonate is a strategy that continues to be employed at our institutions. We did not observe a statistically significant difference in incidence of TLS between patients who did and did not receive preventive IV sodium bicarbonate. Since 2017, the practice at our institutions has been to omit bicarbonate from IV fluids.

\section{Rasburicase for TLS Prevention}

In this study, $14 \%$ of intermediate-risk and $28 \%$ of high-risk patients had elevated serum uric acid within the data collection window, and 3\% of intermediate-risk and 36\% of high-risk patients received preventive rasburicase. It is likely that more of the high-risk patients received preventive rasburicase because they presented with more extreme metabolic derangements. Notably, the pattern of rasburicase use that we observed is inconsistent with clinical guidelines, which currently recommend that all high-risk patients receive initial management with rasburicase for TLS prevention. ${ }^{2,4}$
We did not observe a statistically significant difference in incidence of TLS between patients who did and did not receive preventive rasburicase. The efficacy of rasburicase in reducing serum uric acid in adults at risk for TLS has been well characterized. ${ }^{12-14}$ Studies have investigated the efficacy of fixed- and weight-based dosing in reducing serum uric acid, although whether these dosing regimens produce favourable clinical outcomes (such as reduction in incidence of acute kidney injury and need for RRT) has not been determined. ${ }^{15-19}$ The manufacturer of rasburicase recommends a daily dose of $0.20 \mathrm{mg} / \mathrm{kg}$ for up to 7 days for treatment and prophylaxis of hyperuricemia. ${ }^{20}$ In one study, which compared rasburicase $0.15 \mathrm{mg} / \mathrm{kg}$ administered as a single dose followed by daily as-needed doses with a fixed regimen of 5 daily doses, administration of a single dose produced a sustained decrease in serum uric acid, and few patients who received the single dose required additional doses of rasburicase. ${ }^{21}$ It is standard practice at the authors' institutions to administer $4.5 \mathrm{mg}$ of rasburicase to patients at risk of TLS, so we are unable to comment on whether administration of a weightbased dose would decrease TLS incidence in our setting. The possibility of a specific population that would derive clinical benefit from use of a preventive weight-based dose of rasburicase remains to be elucidated.

Of the 10 patients in our study who received preventive rasburicase, only 4 had a serum uric acid at or above $476 \mu \mathrm{mol} / \mathrm{L}$ at the time of administration. In late 2019, the cost of a single 4.5-mg dose of rasburicase at one of the study institutions was approximately $\$ 410$, and thus judicious prescribing is warranted. Studies investigating the efficacy of rasburicase in reducing serum uric acid generally include patients whose serum uric acid is 
This single copy is for your personal, non-commercial use only.

For permission to reprint multiple copies or to order presentation-ready copies for distribution, contact CJHP at publications@cshp.pharmacy

elevated at the time of presentation. As a result, it is unknown whether there is benefit in administering rasburicase to those with serum uric acid below this threshold. Furthermore, only 1 of the 4 patients who had laboratory TLS on admission and who also had elevated serum uric acid received rasburicase for treatment. Our institutions would benefit from reassessment of rasburicase prescribing practices to ensure that the medication is being used judiciously in the population most likely to derive benefit.

\section{Additional Outcomes}

In this study, TLS developed during admission for $14 \%$ of intermediate-risk and $8 \%$ of high-risk patients. It has been estimated that intermediate-risk patients have a $1 \%-5 \%$ chance of tumour lysis, whereas high-risk patients have a greater than $5 \%$ risk of tumour lysis. ${ }^{3}$ We observed that high-risk patients were more likely than intermediate-risk patients to have a relevant laboratory abnormality, which might have prompted more aggressive management (for example, administration of a higher volume of IV fluids) to decrease the likelihood of TLS.

Strategies employed for the treatment of TLS were similar between those who presented with TLS and those in whom TLS developed during the admission, although a comparative analysis of treatment interventions was not performed because of the small sample size. Only 1 of the 2 patients who presented with TLS and who received 2 doses of rasburicase had persistence of serum uric acid above $476 \mu \mathrm{mol} / \mathrm{L}$ before administration of the second dose. Interestingly, 3 of the 6 patients in whom laboratory TLS developed during admission had serum uric acid above $476 \mu \mathrm{mol} / \mathrm{L}$ yet did not receive rasburicase; 2 of these patients also experienced clinical TLS (in the form of acute kidney injury). The decision to not administer rasburicase in these instances was likely multifactorial and influenced by the acuity of the patient's presentation, in combination with the decision to pursue other management strategies when possible (e.g., RRT in the ICU).

\section{Strengths and Limitations}

To our knowledge, this is the first study to investigate prescribing practices related to TLS prevention strategies in Canada and to associate these practices with clinical outcomes. We included patients from a malignant hematology service and an affiliated ICU in Toronto to reflect inter-institutional prescribing practices. We set specific, evidence-based criteria for inclusion, exclusion, and risk stratification in an effort to maintain uniformity of the study population.

We relied on ICD-10 codes to identify potential participants, under the assumption that patient charts had been accurately and thoroughly coded. The risk of selection bias because of the retrospective study design limits our ability to conclusively assess the impact of preventive strategies on clinical outcomes or to provide specific recommendations for practice.

\section{Future Directions}

Previous studies have demonstrated success in changing practice with interventions such as education and modification of standard order sets. ${ }^{22}$ On the basis of our study findings, we recommended several changes to practice at our own institutions. Hydration with IV sodium bicarbonate should no longer be used for TLS prevention, given its uncertain benefit and potential harms, and our institutions have already discontinued this practice by removing this option from standard order sets. We are currently reviewing prescribing practices for rasburicase, because the pattern of use that we observed neither complies with clinical guidelines nor appears to be associated with a reduction in incidence of TLS. We are investigating strategies for optimization of rasburicase use, such as the use of alternative dosing regimens or the use of this drug in specific patient populations. In general, clinicians and patients would benefit from larger-scale research to better guide TLS risk stratification and management of case for patients with hematologic malignancy.

\section{CONCLUSION}

At the study institutions, almost all patients admitted with hematologic malignancy who were at intermediate or high risk for TLS received IV fluids and allopurinol to prevent TLS. Most patients received IV fluids containing sodium bicarbonate for this purpose, despite guidelines recommending against its use. Preventive rasburicase was more commonly used in patients at high risk, although use of this drug was not universal within this group despite the recommendations of clinical guidelines. Among all patients, there was no difference in TLS incidence between those who did and did not receive preventive IV sodium bicarbonate or rasburicase, although the numbers were small. Higher-quality evidence is needed to guide risk stratification and management for patients at intermediate and high risk of TLS.

References

1. Cairo MS, Bishop M. Tumour lysis syndrome: new therapeutic strategies and classification. Br J Haematol. 2004;127(1):3-11.

2. Coiffier B, Altman A, Pui CH, Younes A, Cairo MS. Guidelines for the management of pediatric and adult tumor lysis syndrome: an evidence-based review. J Clin Oncol. 2008;26(16):2767-78.

3. Cairo MS, Coiffier B, Reiter A, Younes A; TLS Expert Panel. Recommendations for the evaluation of risk and prophylaxis of tumour lysis syndrome (TLS) in adults and children with malignant diseases: an expert TLS panel consensus. Br J Haematol. 2010;149(4):578-86.

4. Jones GL, Will A, Jackson GH, Webb NJA, Rule S. Guidelines for the management of tumour lysis syndrome in adults and children with haematological malignancies on behalf of the British Committee for Standards in Haematology. Br J Haematol. 2015;169(5):661-71.

5. Executive Committee of the Medical Staff. Tumour lysis syndrome (TLS) in adult patients. Version 2. Houston (TX): University of Texas MD Anderson Cancer Center, Department of Clinical Effectiveness; 2018 [cited 2019 Nov 21]. Available from: https://www.mdanderson.org/documents/ for-physicians/algorithms/clinical-management/clin-management-tumorlysis-web-algorithm.pdf

6. Sood AR, Burry LD, Cheng DKF. Clarifying the role of rasburicase in tumor lysis syndrome. Pharmacotherapy. 2007;27(1):111-21.

7. Howard SC, Jones DP, Pui CH. The tumor lysis syndrome. N Engl J Med. 2011;364(19):1844-54. 
This single copy is for your personal, non-commercial use only. For permission to reprint multiple copies or to order presentation-ready copies for distribution, contact CJHP at publications@cshp.pharmacy

8. Adrogué HJ, Rashad MN, Gorin AB, Yacoub J, Madias NE. Assessing acidbase status in circulatory failure. N Engl J Med. 1989;320(20):1312-6.

9. Kim HJ, Son YK, An WS. Effect of sodium bicarbonate administration on mortality in patients with lactic acidosis: a retrospective analysis. PLoS One. 2013;8(6):e65283.

10. Adeva-Andany MM, Fernández-Fernández C, Mouriño-Bayolo D, Castro-Quintela E, Domínguez-Montero A. Sodium bicarbonate therapy in patients with metabolic acidosis. Sci World J. 2014;2014:Article 627673.

11. Conger JD, Falk SA. Intrarenal dynamics in the pathogenesis and prevention of acute urate nephropathy. J Clin Invest. 1977;59(5):786-93.

12. Cortes J, Moore JO, Maziarz RT, Wetzler M, Craig M, Matous J, et al. Control of plasma uric acid in adults at risk for tumor lysis syndrome: efficacy and safety of rasburicase alone and rasburicase followed by allopurinol compared with allopurinol alone-results of a multicenter phase III study. J Clin Oncol. 2010;28(27):4207-13.

13. Pui CH, Jeha $S$, Irwin D, Camitta B. Recombinant urate oxidase (rasburicase) in the prevention and treatment of malignancy-associated hyperuricemia in pediatric and adult patients: results of a compassionate-use trial. Leukemia. 2001;15(10):1505-9.

14. Coiffier B, Mounier N, Bologna S, Fermé C, Tilly H, Sonet A, et al. Efficacy and safety of rasburicase (recombinant urate oxidase) for the prevention and treatment of hyperuricemia during induction chemotherapy of aggressive non-Hodgkin's lymphoma: results of the GRAAL1 (Groupe d'Étude des Lymphomes de l'Adulte Trial on Rasburicase Activity in Adult Lymphoma) study. J Clin Oncol. 2003;21(23):4402-6.

15. McDonnell AM, Lenz KL, Frei-Lahr DA, Hayslip J, Hall PD. Single-dose rasburicase $6 \mathrm{mg}$ in the management of tumor lysis syndrome in adults. Pharmacotherapy. 2006;26(6):806-12.

16. Trifilio SM, Pi J, Zook J, Golf M, Coyle K, Greenberg D, et al. Effectiveness of a single 3-mg rasburicase dose for the management of hyperuricemia in patients with hematological malignancies. Bone Marrow Transplant. 2011; 46(6):800-5.

17. Reeves DJ, Bestul DJ. Evaluation of a single fixed dose of rasburicase $7.5 \mathrm{mg}$ for the treatment of hyperuricemia in adults with cancer. Pharmacotherapy. 2008;28(6):685-90

18. Campara M, Shord SS, Haaf CM. Single-dose rasburicase for tumour lysis syndrome in adults: weight-based approach. J Clin Pharm Ther. 2009; 34(2):207-13.

19. McBride A, Lathon SC, Boehmer L, Augustin KM, Butler SK, Westervelt P. Comparative evaluation of single fixed dosing and weight-based dosing of rasburicase for tumor lysis syndrome. Pharmacotherapy. 2013;33(3):295-303.

20. Fasturtec (rasburicase) [product monograph]. Laval (QC): Sanofi-Aventis Canada Ltd; 2016.

21. Vadhan-Raj S, Fayad LE, Fanale MA, Pro B, Rodriguez A, Hagemeister FB, et al. A randomized trial of a single-dose rasburicase versus five-daily doses in patients at risk for tumor lysis syndrome. Ann Oncol. 2012;23(6):1640-5.

22. Fan-Lun C, Chung C, Lee EHG, Pek E, Ramsden R, Ethier C, et al. Reducing unnecessary sedative-hypnotic use among hospitalised older adults. BMJ Qual Saf. 2019;28(12):1039-45.
Sarah McKenna, PharmD, ACPR, is with the Department of Pharmacy, Mount Sinai Hospital, Sinai Health System, Toronto, Ontario.

Alexandra Cheung, BScPhm, PharmD, is with the Department of Pharmacy, Mount Sinai Hospital, Sinai Health System, Toronto, Ontario.

Amanda Wolfe (formerly Amanda Jacques), BScPharm, ACPR, was, at the time this study was conducted, with the Department of Pharmacy, Princess Margaret Cancer Centre, University Health Network, and the Leslie Dan Faculty of Pharmacy, University of Toronto, Toronto, Ontario. She is now with the Department of Pharmacy, Bruyère Continuing Care, Ottawa, Ontario.

Brenda L Coleman, PhD, is with Infectious Disease Research, Mount Sinai Hospital, Sinai Health System, and the Dalla Lana School of Public Health, University of Toronto, Toronto, Ontario.

Michael E Detsky, MD, MSHP, FRCPC, is with the Interdepartmental Division of Critical Care, University Health Network/Sinai Health System and the Interdepartmental Division of Critical Care and Faculty of Medicine, University of Toronto, Toronto, Ontario.

Laveena Munshi, MD, MSC, FRCPC, is with the Interdepartmenta Division of Critical Care, University Health Network/Sinai Health System, Toronto, Ontario.

Dawn Maze, MD, MSc, FRCPC, is with the Division of Medical Oncology and Hematology, Princess Margaret Cancer Centre, University Health Network, and the Faculty of Medicine, University of Toronto, Toronto, Ontario.

Lisa Burry, PharmD, is with the Department of Pharmacy, Mount Sina Hospital, Sinai Health System, and the Leslie Dan Faculty of Pharmacy, University of Toronto, Toronto, Ontario.

Competing interests: Dawn Maze has received personal fees from Novartis and Pfizer for activities outside the scope of the study reported here. No other competing interests were declared.

\section{Address correspondence to:}

Dr Sarah McKenna

Department of Pharmacy (18-377)

Mount Sinai Hospital, Sinai Health System

600 University Avenue

Toronto ON M5G 1X5

e-mail: sarah.mckenna@sinaihealthsystem.ca

Funding: This project was supported by the Department of Pharmacy at both Mount Sinai Hospital and Princess Margaret Cancer Centre. There was no additional funding source.

Acknowledgements: The authors would like to acknowledge Karen Wang, Justine Del Rosario, Caddie Sze, and Samantha Polito for their assistance with this project. They would also like to acknowledge the Princess Margaret Cancer Registry for assistance with data retrieval. 